\title{
Enzymatic Synthesis of Phenolic CoAs Using 4-Coumarate:coenzyme A Ligase (4CL) from Rice
}

\author{
Yoon-Jung Lee, Youngmin Jeon, Jong Suk Lee, ${ }^{\dagger}$ Bong-Gyu Kim, Choong Hwan Lee, and Joong-Hoon Ahn ${ }^{\circ}$ \\ Deparment of Bioscience and Biotechnologv, Bio. Holectlar Informatics Center, IBST, Konkuk Lniversitw, Seoul 143-701, Korea \\ E-mail: jhahnakonkukackr \\ ${ }^{\dagger}$ Korea Research Institute of Bioscience and Biotechologv (KRIBB), Daejeon 305-333, Korea \\ Received December 4, 2006
}

Key Words : 4-Coumarate:CoA ligase Cimnamoỵl-CoA. Caffeoyl-CoA. Fenuloỵl-CoA. Flavonoid bioșynthesis

Generally. biological synthesis using enzymes or cells has an advantage over chemical synthesis for compounds requiring chirality and regioselectivity. ${ }^{1.2}$ In addition. reaction condition of biological synthesis is much milder and several synthetic steps can be omitted. ${ }^{3.4}$ Thus. recently. many researches have been done to develop suitable biocataly'sis.

During in vitro study on biosynthetic pathway one of the difficulties was to find suitable substrates. For in vitro characterization of enzyme. special forms of intermediates are required. which need complicate reaction scheme for organic synthesis. ${ }^{5}$ Among them. coenzyme A (CoA) thioesters are important intermediates found in many biological processes such as fatty acid metabolism. and citric acid cycle. In the phenylpropanoid pathway leading to flavonoid. anthocyanin and lignin biosynthesis. several phenolic CoA thioesters serve as important substrates. ${ }^{7}$

The enzyme 4-coumarate:CoA ligase (4CL) catalyzes the conversion of 4-coumaric acid into coumaroyl-CoA and a few related substrates into their corresponding products such as cinmamoy $1-\mathrm{CoA}$ caffeoyl-CoA and feruloy $1-\mathrm{CoA}$ in a process utilizing ATP and thus channels the common, phenylalanine-derived building block into the widely distinct branches of general phenylpropanoid metabolism. The phenylpropanoid branch pathways have diverse functional compounds in plant development and environmental interactions. such as lignin for structure support. flavonoids for UV protection. and anthocyanins and chalcones as pigments for the attraction of pollinators. Furthermore. CoA esters made by 4coumarate $\mathrm{CoA}$ ligase (4CL) represent a key class of activated intermediates in these pheny lpropanoid branch pathway.

Currently. we have studied in phenylpropanoid and flavonoid biosynthesis in rice ${ }^{10.11}$ In vitro characterization of enzymes in these pathways has been hindered by the lack of intermediates such of caffeoyl-CoA. However. conventional chemical synthesis of these intermediate compounds required multiple synthetic steps with a low yield. whereas enzymatic synthesis requires just a single step. Thus. we searched for sequences highly homologous to $4 \mathrm{CL}$ from rice (R4CL). Two primers. 5'-GAGATTATGGGGTCGGTGG-3' as a forward primer and 5'-TTTTTCACCGGTTGTGTGAC-3' as a reverse primer were synthesized based on the R4CL sequence (GenBank Accession number BAD27987). $R+C L$ was cloned by polymerase chain reaction with $\mathrm{cDNA}$ as a template and the above two primers. cDNA was synthesized with total RNA isolated from 3 weeks old rice seedling using onuniscript reverse transcriptase (Qiagen. Geman). PCR was carried out with Hotstart Taq polymerase (Qiagen) under the following conditions: 40 cycles of $1 \mathrm{~min}$ denaturation at $94^{\circ} \mathrm{C} .1 \mathrm{~min}$ annealing at $55^{\circ} \mathrm{C}$. and $2 \mathrm{~min}$ amplification at $72^{\circ} \mathrm{C}$. Then. PCR product was subcloned into pGEMT-Easy vector (Promega. USA) and its both strands were sequenced. The open reading frame of $R+C L$ consisted of $1665 \mathrm{bp}$. which is predicted to encode $59.6 \mathrm{kDa}$ protein.

In order to use R4CL for the attaclument of CoA to phenolic compounds, the open reading frame of R4CL was subcloned into Escherichia coli expression vector pGEX $5 X-1$, which contains glutathione $S$-transferase. The resulting construct was transformed into $E$. coli BL21. Subsequently, $E$. coli harboring $R+C L$ cultivated in LB medium containing $50 \mu \mathrm{g} / \mathrm{mL}$ ampicilin. and the culture was allowed to grow until absorbance at $600 \mathrm{~nm}$ reached 0.6 to 0.8 . At this point. IPTG was added at a final concentration of $0.1 \mathrm{mM}$ and the culture was incubated for additional 5 hours at $28^{\circ} \mathrm{C}$. The resulting recombinant protein was purified using glutathione S-transferase affinity colunu. The purified proteins were analyzed by $10 \%$ SDS-polyacrylamide gel electrophoresis. As shown in Figure 1, R4CL was successfully expressed in E. coll and purified to near homogeneity. The R4CL was purified by 10 fold with $8.4 \%$ yield. The molecular weight of the recombinant R4CL was about $86-\mathrm{kDa}$ which corresponds well with the sum of the predicted molecular weight

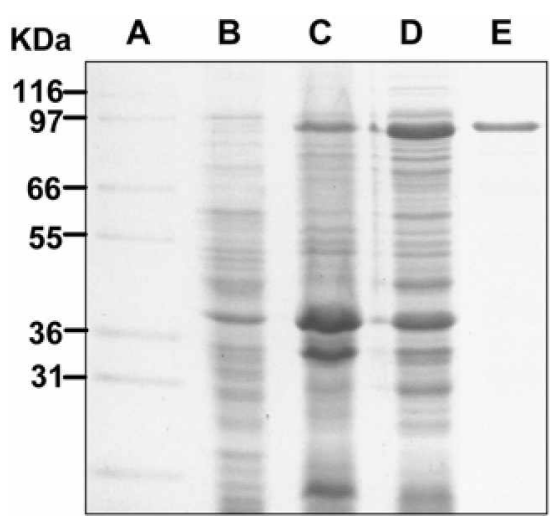

Figure 1. SDS-PAGE analysis of the recombinant R4CL. A, Molecular weight size markers; B, E. coli ly sate before induction: C. E. coli lysate after induction; D, Soluble protein after sonication: $E$, puritied $4 C L$ with GSl attinity column. 
of R4CL (59.6-kDa) and the molecular weight of GST (26 $\mathrm{kDa}$ ).

Enzy'matic sy'nthesis of phenolic CoA was carried out with the purified R+CL. The reaction mixture contained $100 \mathrm{mM}$ Tris- $\mathrm{HCl}$ (pH 7.5). $2.5 \mathrm{mM} \mathrm{MgCl}, 2.5 \mathrm{mM}$ ATP. $100 \mu \mathrm{M}$ coenzyme $\mathrm{A}$ and $100 \mu \mathrm{M}$ substrates (caffeic acid, $p$ coumaric acid ferulic acid. and sinapic acid) in $1 \mathrm{~mL}$ and the reaction was performed at room temperature. The reaction was stopped by $3.5 \%$ trichloroacetic acid in $50 \%$ acetonitrile and the reaction products were analyzed using Varian high performance liquid chromatograply (HPLC: Palo Alto. CA. USA) equipped with $\mathrm{C} 18$ reversed-phase column (Waters Milford. MA. $4.60 \times 250 \mathrm{~mm} .0 .6$ micron) and a photo diode array detector. For analytical scale. the mobile phase consisted of $0.1 \%$ formic acid buffer $(\mathrm{pH} 3.0)$ was programmed as follow: $10 \%$ acetonitrile at $0 \mathrm{~min} .40 \%$ acetonitrile at 10 $\min .70 \%$ acetonitrile at $20 \mathrm{~min}$. and $90 \%$ acetonitrile at 30 min. The flow rate was $1 \mathrm{~mL} / \mathrm{min}$ and UV detection was performed at $340 \mathrm{~mm}$. Analysis of reaction products with HPLC showed that caffeic acid $p$-coumaric acid. and ferulic acid gave new peaks that had different retention time with substrate itself but analysis of sinapic acid reaction product did not show any new peaks. This result indicted that R4CL used caffeic acid. $p$-coumaric acid and ferulic acid as a substrate. Furthenmore liquid chromatography mass spectrometry (LC-MS) was performed using a Finnigan LCQ Advantage MAX ion trap mass spectrometer (Themo Electron Co. Madison. WI. USA) equipped with an electrospray ionization (ESI) source. HPLC separations was performed on the Finnigan Surveyor ${ }^{\mathrm{TM}}$ Modular HPLC Systems (Thermo Electron Co.) using a YMC Hýdrosphere C18 (5 $\mu \mathrm{m} .50 \times 2.0 \mathrm{~mm}$. YMC Co. Kyoto. Japan) with BetaBasic18 guard columun $(2.1 \times 10 \mathrm{~mm}$. Thermo. USA). The system was operated by the Lee $u t$ al. method."

Molecular weight of caffeic acid reaction product was $m z$

A

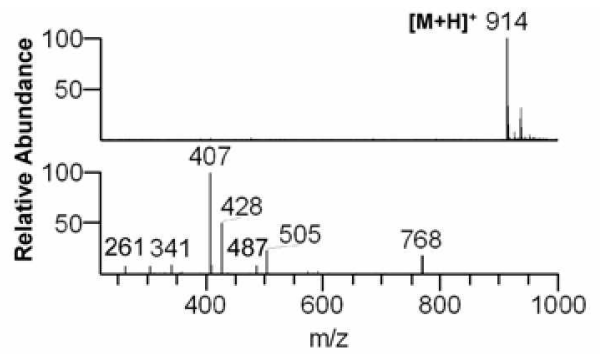

B

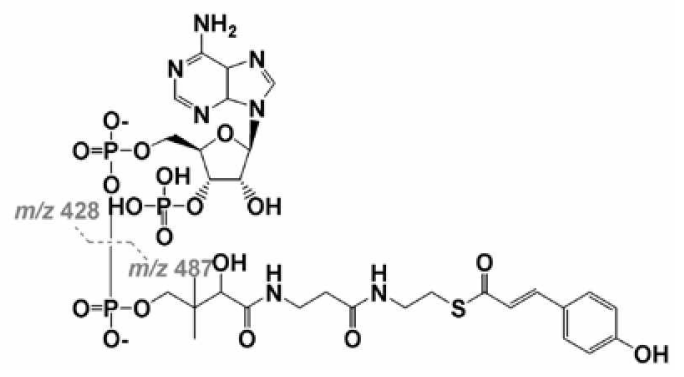

Figure 2. Mass spectrum and MS/MS tragment analysis of the $p$ commaric acid reaction product with R4CL2. A, Mass and MS/MS spectrum of the $p$-commaric acid reaction product: $B$, identified tragments of $p$-coumaroyl CoA by MS/MS analysis.
Table 1. Conversion of R4CL with p-coumaric acid, cafteic acid, and terulic acid

Caffeic acid

Sinapic acid was not serzed as a substrate for R4CL. Fifty $\mu \mathrm{g}$ of the purified R4CL was used with $100, \mu \mathrm{M}$ of each substrate. Reaction misture was incubated at $25^{\circ} \mathrm{C}$ for $12 \mathrm{hrs}$

$930\left([\mathrm{M}+\mathrm{H}]^{+}\right)$based on mass spectrometer, which agreed with the predicted molecular weight (MW 929) of the predicted caffeoyl-CoA (Fig. 2). Also molecular weight of ferulic acid $\left(m z 944 .[\mathrm{M}+\mathrm{H}]^{-}\right)$and $p$-coumaric acid reaction product $\left(m z 914 .[\mathrm{M}+\mathrm{H}]^{-}\right)$agreed well with the predicted molecular weight of the predicted corresponding CoAs. Furthermore, relative reactivity of caffeic acid $p$-coumaric acid and ferulic acid was measured. Among them. R4CL exhibited the highest activities toward $p$-coumaric acid as a substrate. After 12 lurs incubation of $100 \mu \mathrm{M}$ of caffeic acid, $p$-coumaric acid and ferulic acid with $50 \mu \mathrm{g}$ of the purified R4CL at 25 ${ }^{\circ} \mathrm{C} .62 \mu \mathrm{M}$ of caffeoyl-CoA. $45 \mu \mathrm{M}$ of feruloyl-CoA and 66 $\mu \mathrm{M}$ of $p$-coumaroyl-CoA could be obtained (Table 1).

Acknowledgements. This work was supported partially by a grant from Rural Development Administration. Republic of Korea and KRF-2006-005-J0340I (KRF). Yoon Jung Lee was supported partially by the $2^{\text {nd }}$ Brain Korea 21 from Minister of Education, Korea.

\section{References}

1. Raadt. A. D.: Griengl. H. Chr. Opin. Biotechnol. 2002. 13.537

2. Lee. P. H.: Kiml. H.: Lee. K.: Dong. S.: Kim. S.: Kim. H.: Kim. H.: Lee, M.: Shim. E.; Lee, S.: Kim, M.: Han, M.: Noh, K.: Sridhar. M. Bull. Korean Chem. Soc. 2004, 25. 1687.

3. Kim. B. G.: Kim, H.: Kim, J. H.; Lim. Y.: Ahn, J.-H. Bull Konan Chem. Soc. 2006, 27. 357.

4. Kim. J. H.; Kim, B. G.; Park. Y; Han. J. H.; Lim. Y: Ahn. J.-H Agric. Chem. Biotechol 2006. 19.114.

5. Obel. N.: Scheller. H. V. Anal Biochem. 2000. 286.38.

6. Lehninger. A. L.: Nelson. D. L.: Cox. M. M. Principles of Biochemistry, 2nd ed.: Worth Publishers: New York, 1993; p 380.

7. Croteau. R.: Kutchan. T. M.: Lewis. N. G. It Natural Products: Biochentistry and Holecular Biology of Plants in Natural Product: Buchanan. B. B.: Gruissem. W.: Iones. R. L.. Eds.: American Society of Plant Physiologists: Rockille. 2000: p 1250.

8. Douglas. C. T. Trends Plont Sci. 1996. 1. 171

9. Dixon, R. A.: Paiva. N. L. Plant Cell 1995, 7, 1085.

10. Kim, B. G.: Lee. Y.: Hur, H.-G.: Lim. Y; Ahn. J.-H Plotochenisty 2006. 67,387

11. Ko. J. H.: Kim. B. G.: Hur. H.-G.: Limn. Y.: Ahn. I.-H. Plam Cell Rep. 2006. 25. 141 .

12. Lee. I. S.: Kim. D. H.: Liu. K.-H.: Oh. T. G.: Lee. C. H. Rapid Commun. Mass Spectron. 2005. 19,3539. 